\title{
Development of an Optimal Methodology to Solve the Restoration Problem in Electricity Distribution Networks
}

\section{Bárbara R. Rosado*, Marcos J. Rider}

\begin{abstract}
The restoration of electricity distribution networks determines the switching operations needed to isolate a faulted area, aiming to minimize operating costs related to the disconnection of loads and to the number of switching operations. Additionally, the final topology after the system restoration must ensure that the operational constraints are respected. In other words, the limits of nodal voltage and branch current magnitudes, the feeders capacities and the radial operation of the distribution networks must be respected. In this context, this work aims to develop modern convex models to solve the problem of restoration in electricity distribution networks. These models bring robustness, generality, and flexibility. Moreover, they are efficiently solved using commercial computational solvers, which makes them interesting for utilities.
\end{abstract}

\section{Key words: \\ Mathematical Modeling. Optimization. Restoration of Distribution Networks.}

\section{Introduction}

The restoration of electrical networks, considering the potential of intelligent networks, can improve the operational efficiency and provide significant reductions in Middle Time of Emergency Response. It results in a reduction of fines generated by noncompliance with the requirements established by the National Electric Energy Agency (ANEEL in Portuguese) ${ }^{1}$. After the occurrence of a permanent fault in distribution networks, restoration schemes are used to restore electrical service in network areas that have been disconnected due to a remote fault, by the operation of switches or breakers allocated along the feeders. The network topology can be modified to isolate the area directly affected by the fault and to restore the maximum possible amount of demand ${ }^{2}$ by transferring the load among the other feeders, respecting the operating limits. This is a decision-making problem, it is complex and difficult to solve due to the combinatorial nature of the problem and the non-linearity in the operating constraints of distribution networks. Because of that, there are few feasible solutions and much time can be required to solve it. Currently, the utilities are interested in solving the restoration problem efficiently, reliably and quickly, considering they use some outdated practice. It is important to obtain a quick solution because the utilities have only 3 minutes $^{1}$ before starting to pay fees due to the de-energized zones. In this context, this work proposes a modern methodology that solves the restoration problem quickly and recovers the maximum number of zones with minimum switching. The methodology is developed using a mixed-integer linear problem model (MILP) that describes and solves the restoration problem.

\section{Results and Discussion}

The distribution network from Barão Geraldo, Campinas, is used to demonstrate the applicability of the developed method. The network has 14 feeders, 5293 nodes, 5256 circuits, and 34 switches. Image 1 shows the network in the moment a fault happens. Image 2 illustrates the final configuration obtained by the methodology developed. In Image 2, one can see that the faulted zone is isolated by opening the switches. Besides, the zones connected to the faulted zone were transferred to other feeders by closing the switches identified in Image 2.

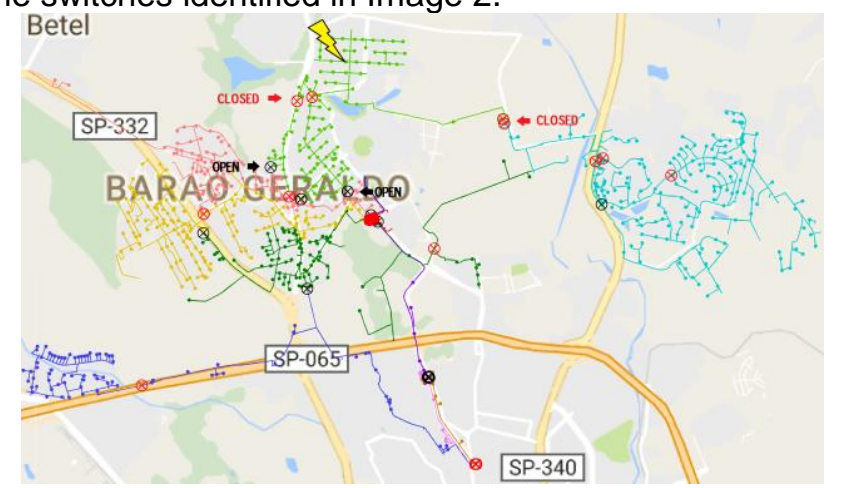

Image 1. Electrical system immediately when a fault happens. Betel

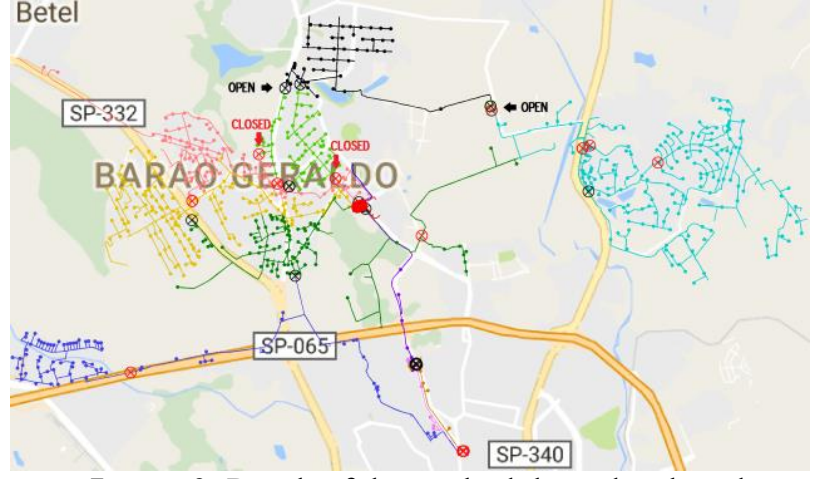

Image 2. Result of the methodology developed.

All zones were recovered (colored grid) after isolating the faulted zone (black grid).

\section{Conclusions}

This work presents a modern methodology that describes and solves the restoration problem. As it provides real-time solutions, the tool can be used by utilities not only to automate the control of the system but also to train the operators and to improve the practice utilized.

\section{Acknowledgement}

This work was supported by a São Paulo Research Foundation (FAPESP) grant \#2016/10010-1.

\footnotetext{
National Electric Energy Agency

ANEEL.

Available:

$<$ http://www.aneel.gov.br> [in Portuguese].
2 Willis HL. Power distribution planning ref

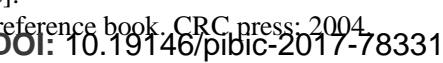

